\title{
Das Strukturierte Interview für Anorektische und Bulimische Ess-Störungen nach DSM-IV und ICD-10 zur Expertenbeurteilung (SIAB-EX) und dazugehöriger Fragebogen zur Selbsteinschätzung (SIAB-S)
}

\author{
M. Fichter ${ }^{a, b} \quad$ N. Quadflieg ${ }^{a}$ \\ a Psychiatrische Universitätsklinik München \\ ${ }^{b}$ Medizinisch-Psychosomatische Klinik Roseneck, Prien
}

\section{Schlüsselwörter}

Ess-Störungen - Anorexia nervosa - Bulimia nervosa .

Binge Eating Disorder - Strukturiertes klinisches Interview

\section{Zusammenfassung}

Hintergrund: Das Strukturierte Inventar für Anorektische und Bulimische Ess-Störungen nach DSM-IV und ICD-10 - bestehend aus dem Interview zur Expertenbeurteilung (SIAB-EX) und dem dazugehörigen Fragebogen zur Selbsteinschätzung (SIABS) - erfasst Ess-Störungs-Symptome und andere, häufig damit einhergehende Symptome wie Ängste, Depression, Zwangssymptome, Substanzabusus und Beeinträchtigung der Sexualität und der sozialen Integration. Der Fragebogen ist parallel zu dem Experteninterview formuliert - allerdings mit allgemeinverständlichen Items. Getrennte Faktorenanalysen ergaben ähnliche Faktorenstrukturen für Fragebogen und Interview, sowie für die beiden erfassten Messpunkte «jetzt» (letzte 3 Monate vor Interview) und "früher» (Pubertät bis zum Zeitpunkt 3 Monate vor dem Interview). Beide Verfahren erlauben sowohl die Diagnose von Ess-Störungen nach DSM-IV und ICD-10, als auch die Berechnung von Summenwerten, welche den generellen Schweregrad einer Ess-Störung charakterisieren. Methode: Die Testgütekriterien wurden an einer Stichprobe von 377 stationär wegen Ess-Störungen behandelten Patienten überprüft. Normative Daten zum SIAB-EX wurden für 202 jungen Frauen ohne Ess-Störungen aus der Normalbevölkerung erhoben. Ergebnisse: Die Testgütekriterien ergaben zufriedenstellende bis sehr gute Ergebnisse. Die Interrater-Reliabilität $(\kappa)$ für das Interview SIAB-EX betrug 0,81 (jetzt) bzw. 0,85 (früher). Sensitivität, Spezifität und "positive predictive value» waren sehr gut für SIAB-EX (PPV = 0,91 für lifetime) und SIAB-S (PPV = 0,98 lifetime). Es werden Mittelwerte für SIAB-EX und SIAB-S für die klinische Stichprobe berichtet. Des Weiteren sind zum SIAB-EX neben den Daten zu essgestörten Patienten und Patientinnen auch normative Daten von 202 jungen, nicht essgestörten Frauen aus der Normalbevölkerung angegeben. Schlussfolgerung: Das Experteninterview SIAB-EX ist geeignet, den "Gold-Standard" für die Erfassung von Ess-Störungen im deutschen Sprachraum darzustellen. Das Interview existiert auch in eng lischer, italienischer und spanischer Sprache.

\begin{tabular}{ll}
\hline KARGER & ( 2001 S. Karger GmbH, Freiburg \\
$\begin{array}{l}\text { Fax +49761 45207 14 } \\
\begin{array}{l}\text { E-mail Information@Karger.de } \\
\text { www.karger.com }\end{array}\end{array}$ & $\begin{array}{l}\text { Accessible online at: } \\
\text { www.karger.com/journals/ver }\end{array}$ \\
&
\end{tabular}

\author{
Key Words \\ Eating disorders - Anorexia nervosa - Bulimia nervosa \\ Binge eating $\cdot$ Structured clinical interview
}

\section{Summary \\ The Structured Interview (SIAB-EX) and Questionnaire} (SIAB-S) for Anorexic and Bulimic Eating Disorders for DSM-IV and ICD-10

Background: The Structured Inventory for Anorexic and Bulimic Syndromes according to DSM-IV and ICD-10 consists of the Structured Expert Interview for Anorexic and Bulimic Syndromes (SIAB-EX) and the corresponding self report questionnaire (SIAB-S). These instruments assess symptoms of eating disorders and other symptoms often found in eating-disordered individuals (e.g. anxieties, symptoms of OCD, depression, substance abuse and impairment of sexuality and social integration). Thus, parallel forms for self-report and expert rating are available. Separate factor analyses resulted in very similar factor structures for self-report and interview as well as for both time points assessed: "current" (last 3 months before the interview) and "past" (time from puberty up to 3 months before the interview). Both assessments can be used for diagnosing eating disorders according to DSM-IV and ICD-10 and a total score can be computed. Method: Test criteria were assessed using a sample of 377 inpatients treated for an eating disorder. For the expert interview, SIAB-EX data were collected in a community sample of 202 young women without eating disorders. Results: Test criteria were satisfying or better. Interrater reliability $(\kappa)$ for the expert interview SIAB-EX was 0,81 (current) and 0,85 (past). Sensitivity, specificity, and positive predictive value were very good for the SIAB-EX (ppv $=0.91$ lifetime) and the SIAB-S (ppv $=0.98$ lifetime). Means for SIAB-EX and SIAB-S for the inpatient sample are reported. For the expert interview SIAB-EX norms for 202 young non-eating disordered women are given. Conclusion: The expert interview SIAB-EX can be seen as a 'gold standard' for the assessment of eating disorders. The SIAB-EX is available in German, English, Italian and Spanish. 


\section{Einleitung}

Über die vergangenen Dekaden haben Ess-Störungen in Industrienationen zugenommen. Sie finden sich besonders häufig, aber nicht ausschließlich, bei Mädchen und jungen Frauen im Alter zwischen 15 und 35 Jahren. Bei der bestehenden Verbreitung von Ess-Störungen ist es wichtig, über Selbst- und Fremdeinschätzungsskalen zu verfügen, die Einsatz finden können beim Screening von Ess-Störungen in größeren Stichproben und die geeignet sind zur Diagnostik von Ess-Störungen und für die «Outcome-Forschung».

In der diagnostischen Klassifikation von Ess-Störungen haben in den letzten Jahrzehnten deutliche Präzisierungen und Differenzierungen stattgefunden. Anorexia nervosa und Bulimia nervosa sind seit mehr als 20 Jahren im Diagnostischen und Statistischen Manual Psychischer Störungen (DSM-IV) der American Psychiatric Association (APA) [deutsch Saß et al., 1996] und seit etwa 10 Jahren auch in der Internationalen Klassifikation von Krankheiten (ICD-10) [Dilling et al., 1991] dokumentiert. Zur «Binge-Eating-Störung» sind im Anhang des DSM-IV vorläufige Kriterien formuliert. Diese Diagnosesysteme basieren auf der operationalisierten Diagnostik, wobei für jede psychische Störung Ein- und Ausschlusskriterien sowie häufig auch Festlegungen zur Zeitdauer und/oder Häufigkeit von Symptomen angegeben werden.

Eine valide und reliable Erfassung von Ess-Störungs-Symptomen ist Voraussetzung für die Anwendung der oben erwähnten Diagnosesysteme. Dies ist für die Erforschung der medizinischen, psychologischen und biologischen Aspekte von EssStörungen unentbehrlich. Dabei reicht das Spektrum der zu berücksichtigenden Symptome von Ess-Störungs-spezifischen wie Gewichtsphobie, Nahrungsvermeidung oder Essattacken bis zu eher unspezifischen Bereichen der Psychopathologie wie Depression, Angst oder Einschränkungen im Selbstwertgefühl oder in der sozialen Kompetenz und Partnerschaft.

Um die Symptome von Ess-Störungen angemessen zu erfassen, sind standardisierte Instrumente erforderlich. Zu diesem Zweck wurden mehrere Selbstauskunfts-Verfahren entwickelt. Unter anderem sind dies das Eating Disorder Inventory [Garner et al., 1983, Garner, 1991], das Three Factor Eating Questionnaire [Stunkard und Messick, 1985; deutsch Fragebogen zum Essverhalten, Pudel und Westenhöfer, 1989], und der Eating Attitudes Test [Garner und Garfinkel, 1979]. Von Cooper und Fairburn [1987] stammt das Experten-Interview Eating Disorder Examination (EDE) zur Erfassung der EssStörungs-Pathologie, welches im englischsprachigen Raum häufig benutzt wird. Fairburn und Beglin [1994] fanden Diskrepanzen zwischen Selbstauskünften Betroffener und der Einschätzung durch klinische Experten bei Angaben zu den Essattacken, wobei die Selbstauskünfte höhere Raten ergaben. Andererseits wurden keine Unterschiede bei weniger komplexen Verhaltensweisen wie Erbrechen oder Laxantienabusus festgestellt. Black und Wilson [1996] bestätigten diese Befunde bei Frauen, die einen Substanzabusus aufwiesen und berichteten von einer Überschätzung der Probleme bezüglich Figur und Gewicht durch Selbstauskünfte. Greeno et al. [1995] verglichen die Binge Eating Scale (Selbstauskunft) mit der EDE und betonten die begrenzte Präzision von Selbstauskünften. Generell werden in der aktuellen psychiatrischen Forschung standardisierte oder halbstrukturierte Experteninterviews als der «Gold-Standard» angesehen, ohne den Wert eines Selbstauskunftsfragebogens als Screening-Instrument in Frage zu stellen.

Parallel zur Entwicklung der englischsprachigen EDE (derzeit aktuelle Version ist die 12. Auflage [Fairburn und Cooper, 1993]) haben wir mit etwas anderer Zielsetzung das «Strukturierte Interview für Anorektische und Bulimische Ess-Störungen nach DSM-IV und ICD-10 zur Expertenbeurteilung (SIAB-EX) mit dem dazugehörigen Fragebogen zur Selbsteinschätzung (SIAB-S)» entwickelt. Das Instrumentarium wurde 1999 im Hogrefe-Verlag veröffentlicht und ist von dort zu beziehen. Die EDE liegt nicht in einer offiziellen Übersetzung in deutscher Sprache vor. Das SIAB-EX unterscheidet sich von der EDE in mehreren bedeutsamen Punkten und geht weit über das von der EDE erfasste Spektrum hinaus. So erfasst das SIAB-EX nicht nur die Leitsymptome der Ess-Störungen, sondern sämtliche relevanten Störungsbereiche essgestörter Patienten. Das SIAB-EX wird derzeit in einer groBen internationalen Multi-Center-Studie über die Genetik von Ess-Störungen zur definitiven Festlegung des Phänotyps der Ess-Störung verwendet [Halmi et al., 2000; 2001; Kaye et al., 2000; Klump et al., 2000].

Die vorliegende Arbeit soll sowohl das Experten-Interview SIAB-EX als auch das Selbstauskunftsverfahren SIAB-S in deutscher Sprache vorstellen, die empirisch gefundenen Faktorenstrukturen aufzeigen und über wichtige statistische Merkmale der beiden Verfahren berichten.

\section{Materialien und Methodik}

Beschreibung des SIAB-EX und SIAB-S

Die 3. Revision des Strukturierten Interviews für anorektische und bulimische Ess-Störungen (SIAB-EX) sowie die Selbstauskunft SIAB-S umfassen jeweils 87 Fragen. Ein Teil der Fragen besteht aus mehreren Teilfragen. Die Item-Nummern bezeichnen in beiden Versionen die selben Inhalte. Die meisten Fragen werden auf einer Schweregradskala von 0 bis 4 kodiert, wobei die Skala generell folgendermaßen definiert ist:

$0=$ Symptom/Problem nicht vorhanden,

$1=$ Symptom/Problem leicht oder selten vorhanden,

2 = Symptom/Problem deutlich oder öfter vorhanden,

$3=$ Symptom/Problem stark oder häufig vorhanden,

4 = Symptom/Problem sehr stark oder sehr häufig vorhanden.

Des Weiteren liegen Fragen vor, welche zur Diagnostik nach ICD-10 oder DSM-IV benötigt werden. Die Fragen erfassen zwei Zeiträume. Zum einen wird die Symptomausprägung in den letzten 3 Monaten (Jetzt-Zustand) kodiert, zum anderen die Zeit vor den letzten 3 Monaten (früher). Jede Frage des SIAB-EX besteht aus einer Hauptprobe, welche immer gefragt werden muss, sowie Zusatzfragen, welche bei Unklarheiten zum Einsatz kommen. Für Jugendliche liegen angepasste Formulierungen vor, die von Frau Prof. Beate Herpertz-Dahlmann entworfen und getestet 


\begin{tabular}{|c|c|c|c|c|c|c|}
\hline & \multicolumn{2}{|c|}{$\begin{array}{l}\text { Gesamt } \\
(\mathrm{n}=377)\end{array}$} & \multicolumn{2}{|c|}{$\begin{array}{l}\text { Anorexia nervosa } \\
(\mathrm{n}=60)\end{array}$} & \multicolumn{2}{|c|}{$\begin{array}{l}\text { Bulimia nervosa } \\
(\mathrm{n}=97)\end{array}$} \\
\hline & $\mathrm{n}$ & $\mathrm{M}(\mathrm{SD})$ & $\mathrm{n}$ & $\mathrm{M}(\mathrm{SD})$ & $\mathrm{n}$ & $\mathrm{M}(\mathrm{SD})$ \\
\hline \multicolumn{7}{|l|}{ SIAB-EX früher } \\
\hline I Körperschema und Schlankheitsideal & 370 & $1,9(0,8)$ & 60 & $2,6(0,4)$ & 95 & $2,1(0,6) * *$ \\
\hline II Allgemeine Psychopathologie & 370 & $1,5(0,7)$ & 60 & $1,5(0,7)$ & 95 & $1,8(0,8)$ \\
\hline III Sexualität und Soziale Integration & 368 & $2,3(0,8)$ & 60 & $2,7(0,6)$ & 95 & $2,5(0,7)$ \\
\hline IV Bulimische Symptome & 369 & $2,4(1,2)$ & 60 & $1,9(1,4)$ & 95 & $3,4(0,5) * *$ \\
\hline V Gegensteuernde Maßnahmen, Fasten, Substanzmissbrauch & 367 & $1,0(0,6)$ & 60 & $1,0(0,5)$ & 95 & $1,2(0,6)$ \\
\hline VI Atypische Essanfälle & 84 & $1,1(1,1)$ & 14 & $0,4(0,7)$ & 25 & $1,6(1,0)^{* *}$ \\
\hline Gesamtwert & 370 & $1,7(0,5)$ & 60 & $1,9(0,4)$ & 95 & $2,0(0,5)$ \\
\hline \multicolumn{7}{|l|}{ SIAB-EX jetzt } \\
\hline I Körperschema und Schlankheitsideal & 370 & $1,2(0,6)$ & 60 & $1,9(0,4)$ & 95 & $1,3(0,5)^{* *}$ \\
\hline II Allgemeine Psychopathologie und Soziale Integration & 370 & $1,1(0,7)$ & 60 & $1,3(0,6)$ & 95 & $1,3(0,7)$ \\
\hline III Sexualität & 368 & $2,1(1,3)$ & 60 & $3,0(1,0)$ & 94 & $2,0(1,3) * *$ \\
\hline IV Bulimische Symptome & 369 & $1,9(1,2)$ & 60 & $1,8(1,3)$ & 94 & $3,2(0,5) * *$ \\
\hline V Gegensteuernde Maßnahmen, Fasten, Substanzmissbrauch & 369 & $0,4(0,3)$ & 60 & $0,5(0,3)$ & 94 & $0,5(0,4)$ \\
\hline VI Atypische Essanfälle & 86 & $0,8(1,0)$ & 14 & $0,3(0,5)$ & 25 & $1,2(1,0) * *$ \\
\hline Gesamtwert & 370 & $1,2(0,5)$ & 60 & $1,4(0,4)$ & 95 & $1,4(0,4)$ \\
\hline
\end{tabular}

$\mathrm{M}=$ Mittelwert; $\mathrm{SD}=$ Standardabweichung.

** $\mathrm{t}$-Test A. nervosa versus B. nervosa: $\mathrm{p}<0,01$.

wurden. Die Fragen des SIAB-S sind einfacher formuliert und der Verwendung in einem Fragebogen angepasst. Aus dem Maximum des JetztZustands und des Früher-Zustand kann ein Lifetime-Wert («worst ever») errechnet werden. Als besonders hervorzuhebendes Merkmal des Experten-Interviews SIAB-EX ist auf das Vorliegen eines sehr ausführlichen Manuals hinzuweisen, mit detaillierten Anweisungen und Beispielen zur Kodierung der Items.

Das SIAB-EX und der SIAB-S können bei allen Personen durchgeführt werden, bei denen der Verdacht auf eine Ess-Störung vorliegt. Sie können aber auch zur Diagnostik von Ess-Störungen im Rahmen epidemiologischer Studien verwendet werden, insbesondere bei der vorgeschalteten Verwendung des SIAB-S zur Identifikation von Risikoprobanden, bei denen eine erhöhte Wahrscheinlichkeit für eine Ess-Störung besteht. Der SIAB-S ist geeignet, als Grundlage für die Vorbereitung eines Erstinterviews zu dienen, z.B. indem er vor dem ersten persönlichen Kontakt per Post dem prospektiven Klienten zugestellt wird und von diesem zurückgeschickt oder zum Erstgespräch mitgebracht wird. In ähnlicher Weise kann der SIAB-S auch zur Baseline-Bestimmung, sowie - daran anschlieBend - zur Verlaufsevaluation in klinischen Studien oder während einer Therapie (etwa zur Qualitätssicherung und Verlaufsdokumentation) angewendet werden. Mit Hilfe des SIAB-S ist auch eine vereinfachte Diagnostik nach DSM-IV und ICD-10 möglich, da der Fragebogen die gleichen Problembereiche erfasst wie das Interview.

SIAB-EX und SIAB-S ermöglichen eine Auswertung von zwei verschiedenen Ergebnis-Typen. Zum einen können Summenwerte für empirisch fundierte Subskalen berechnet werden (siehe unten), zum anderen können Ess-Störungs-Diagnosen nach DSM-IV und ICD-10 erstellt werden. Bei der Erstellung von Diagnosen wird für das SIAB-EX grundsätzlich bei den von 0 bis 4 kodierten Items ein Wert von 2 bis 4 als das Kriterium erfüllend angesehen, bei der SIAB-S Werte von 1 bis 4, sofern ein Kriterium nicht nur durch die Kodierung von 2 bis 4 erfüllt sein kann, wie z.B. bei der Häufigkeit der Essanfälle. Für die Auswertung der Summenwerte und für die Erstellung von Diagnosen sind Computeralgorithmen erhältlich.

\section{Design und Zusammensetzung der Stichprobe}

Die klinische Stichprobe umfasste 377 Patienten (11 Männer, 366 Frauen), die konsekutiv in der Medizinisch-Psychosomatischen Klinik Roseneck in Prien am Chiemsee wegen einer Ess-Störung behandelt wurden. Sämtliche Daten wurden kurz nach der Aufnahme erhoben. Das mittlere Alter betrug 29,1 \pm 9,3 Jahre (Mittelwert \pm Standardabweichung). Der Body Mass Index (BMI) betrug bei Aufnahme im Mittel 25,7 \pm 11,7. 60 Patienten wiesen bei Aufnahme eine Anorexia nervosa und 97 eine Bulimia nervosa nach DSM-IV auf. 80 Patienten der klinischen Stichprobe (77 Frauen und 3 Männer) wurden mit dem SIAB-EX und der EDE interviewt. Die Interviews von 31 Frauen wurden auf Video aufgenommen und zur Bestimmung der Interrater-Reliabilität herangezogen.

Die Zufalls-Stichprobe von jungen Frauen aus der Normalbevölkerung umfasste 244 Frauen. Die Grundgesamtheit stellten alle Frauen im Alter zwischen 18 und 30 Jahren dar, die im Münchner Stadtteil Laim beim Einwohnermeldeamt zu einem bestimmten Stichtag gemeldet waren. Der Stadtteil Laim wurde gewählt, da er bezüglich der Verteilung der Kennwerte Geschlecht, sozioökonomischer Status und Ausländeranteil repräsentativ für das gesamte Stadtgebiet München ist. Alle Ausländerinnen wurden vor der Erstellung der Liste durch das Einwohnermeldeamt herausgenommen, um Sprachprobleme bei der Durchführung des Interviews zu vermeiden. Von den 244 befragten Frauen wiesen 42 eine Anorexia nervosa, Bulimia nervosa, Binge Eating Disorder oder eine nicht näher bezeichnete Ess-Störung nach DSM-IV irgendwann in ihrem Leben (lifetime) auf, so dass sich eine Stichprobe von 202 Frauen ohne Ess-Störung ergab. Diese waren bei der Befragung im Mittel 25,3 $\pm 4,0$ Jahre alt und wiesen einen BMI von 22,0 \pm 3,6 auf. Der niedrigste BMI (lifetime) war $19,8 \pm 2,5$, der höchste $23,4 \pm 4,6.4$ Frauen $(1,9 \%)$ hatten bei Befragung einen BMI unter 17,5 (lifetime waren dies $24(11,5 \%)$ ), 7 Frauen $(3,4 \%)$ einen BMI über 30 (lifetime $11(5,3 \%)$ ).

\section{Statistische Auswertung}

Um Auskunft über die Subskalenstruktur zu erhalten, wurden mit den Daten der klinischen Stichprobe $(\mathrm{n}=377)$ Hauptkomponenten-Analysen 


\begin{tabular}{|c|c|c|c|c|c|c|c|}
\hline & & \multicolumn{2}{|c|}{$\begin{array}{l}\text { Gesamt } \\
(\mathrm{n}=377)\end{array}$} & \multicolumn{2}{|c|}{$\begin{array}{l}\text { Anorexia nervosa } \\
(\mathrm{n}=60)\end{array}$} & \multicolumn{2}{|c|}{$\begin{array}{l}\text { Bulimia nervosa } \\
(\mathrm{n}=97)\end{array}$} \\
\hline & & $\mathrm{n}$ & $\mathrm{M}(\mathrm{SD})$ & $\mathrm{n}$ & $\mathrm{M}(\mathrm{SD})$ & $\mathrm{n}$ & $\mathrm{M}(\mathrm{SD})$ \\
\hline \multicolumn{8}{|c|}{ SIAB-S früher } \\
\hline I & Bulimische Symptome & 369 & $2,1(1,2)$ & 60 & $1,7(1,2)$ & 95 & $2,9(1,0)^{* *}$ \\
\hline & Allgemeine Psychopathologie & 370 & $1,8(0,9)$ & 60 & $1,7(0,9)$ & 95 & $2,1(0,9)$ \\
\hline & Schlankheitsideal & 369 & $2,3(0,9)$ & 60 & $2,3(0,9)$ & 95 & $2,6(0,8)$ \\
\hline IV & Sexualität und soziale Integration & 368 & $1,7(1,0)$ & 60 & $1,7(0,9)$ & 95 & $2,0(0,9)$ \\
\hline $\mathrm{V}$ & Körperschema & 370 & $1,3(0,8)$ & 60 & $1,8(0,6)$ & 95 & $1,5(0,8)^{* *}$ \\
\hline VI & $\begin{array}{l}\text { Gegensteuernde Maßnahmen, Substanzmissbrauch, Fasten } \\
\text { und Autoaggression }\end{array}$ & 367 & $0,6(0,6)$ & 60 & $0,5(0,5)$ & 95 & $0,8(0,8)^{* *}$ \\
\hline VII & Atypische Essanfälle & 84 & $1,6(1,1)$ & 14 & $0,8(0,8)$ & 25 & $2,4(0,9) * *$ \\
\hline Ges & amtwert & 370 & $1,6(0,7)$ & 60 & $1,6(0,6)$ & 95 & $2,0(0,6) * *$ \\
\hline \multicolumn{8}{|c|}{$S I A B-S$ jetzt } \\
\hline I & Allgemeine Psychopathologie und soziale Integration & 370 & $1,5(0,7)$ & 60 & $1,7(0,7)$ & 95 & $1,7(0,7)$ \\
\hline II & Bulimische Symptome & 369 & $2,0(1,2)$ & 60 & $1,7(1,3)$ & 94 & $3,0(0,8) * *$ \\
\hline III & Körperschema und Schlankheitsideal & 370 & $1,7(0,8)$ & 60 & $2,0(0,8)$ & 95 & $2,0(0,8)$ \\
\hline IV & Sexualität und Körpergewicht & 370 & $1,5(1,0)$ & 60 & $2,7(0,7)$ & 95 & $1,4(0,9)^{* *}$ \\
\hline \multicolumn{8}{|c|}{ V Gegensteuernde Maßnahmen, Fasten und } \\
\hline & Substanzmissbrauch & 369 & $0,4(0,3)$ & 60 & $0,4(0,3)$ & 94 & $0,5(0,4)$ \\
\hline VI & Atypische Essanfälle & 86 & $1,4(1,1)$ & 14 & $0,8(1,0)$ & 25 & $2,0(1,0)^{* *}$ \\
\hline Ges & amtwert & 370 & $1,4(0,5)$ & 60 & $1,6(0,5)$ & 95 & $1,7(0,5)$ \\
\hline
\end{tabular}

$\mathrm{M}=$ Mittelwert; SD = Standardabweichung.

** t-Test A. nervosa versus B. nervosa: $\mathrm{p}<0,01$.

mit Varimax-Rotation gerechnet und zwar getrennt für SIAB-EX und SIAB-S und jeweils getrennt für den Zustand jetzt und früher. Ansonsten werden die üblichen deskriptiven Verfahren verwendet. Die Ergebnisse zu den Testgütekriterien wurden anhand der Daten der klinischen Stichprobe gewonnen.

\section{Ergebnisse}

Ergebnisse der Faktorenanalysen

Für das Experteninterview SIAB-EX ergaben Hauptkomponentenanalysen mit Varimax-Rotation sowohl für den Jetzt-Zustand als auch für den Früher-Zeitraum 6 Faktoren (Tab. 1). Einzelheiten zur Konstruktion finden sich in Fichter et al. [1991; 1998]. Die jeweiligen 6-Faktoren-Lösungen erklärten nach Rotation 42,8\% (früher) bzw. 40,5\% (jetzt) der Varianz. Die analogen Items des SIAB-S wurden ebenfalls mittels Hauptkomponentenanalysen mit Varimax-Rotation überprüft. Da die Items zu den atypischen Essanfällen erst vor kurzem dem SIAB angefügt worden waren, wurden diese wegen der geringen Fallzahl nicht in die Auswertung einbezogen. Für die Früher-Symptomausprägung erschien nach der Rotation eine 6-Faktoren-Lösung am besten interpretierbar zu sein (Tab. 2 oben). 48\% der Varianz wurden durch die 6 Faktoren erklärt. Ein 7. Faktor mit den Items zu den atypische Essanfällen wurde hinzugefügt. Für die Jetzt-Ausprägung war eine 5-Faktoren-Lösung (40,7\% Varianz-Erklärung) am geeignetsten (Tab. 2 unten), wobei ein 6. Faktor (atypische Essanfälle) zusätzlich definiert wurde. Einzelheiten zur Konstruktion finden sich in Fichter und Quadflieg [1999; 2000]. Es ist auch möglich, für die Erstellung von Subskalen des SIAB-S die Faktorenstruktur des Experteninterviews SIABEX heranzuziehen. Damit ist die Möglichkeit gegeben, die Übereinstimmung zwischen der Experten- und der Selbsteinschätzungsform des SIAB zu überprüfen. Näheres dazu ist in Fichter und Quadflieg [1999; 2000] beschrieben. Generell zeigen sich bei den paarweisen Vergleichen mittlere Zusammenhänge zwischen den Experten- und den Selbsteinschätzungen (Korrelationen zwischen 0,3 und 0,6).

\section{Objektivität}

Sowohl zum SIAB-EX als auch zum SIAB-S gibt es genaue Anweisungen zur Erstellung der Diagnosen sowie zur Berechnung der Summenwerte. Das Manual für das SIAB-EX gibt ausführliche Hinweise zu den Fragen mit deskriptiven Definitionen der Skalierung sowie Hinweise zur Befragungstechnik. Demnach kann davon ausgegangen werden, dass die Durchführung, Auswertung und Interpretation unabhängig von der jeweils tätigen Person ist.

\section{Reliabilität}

Interraterreliabilität: Ein großer Vorteil des SIAB-EX liegt darin, dass - im Gegensatz zu allen anderen Experteninter- 
Tab. 3. Korrelationen der SIAB-Subskalen (Jetzt-Zustand) mit den Subskalen des Experten-Interviews Eating Disorder Examination (EDE)

\begin{tabular}{|c|c|c|c|c|c|c|c|}
\hline & \multicolumn{7}{|l|}{ SIAB-EX $(\mathrm{n}=80)$} \\
\hline & $\begin{array}{l}\text { Körperschema } \\
\text { und } \\
\text { Schlankheits- } \\
\text { ideal }\end{array}$ & $\begin{array}{l}\text { Allgemeine } \\
\text { Psychopatho- } \\
\text { logie und soziale } \\
\text { Integration }\end{array}$ & Sexualität & $\begin{array}{l}\text { Bulimische } \\
\text { Symptome }\end{array}$ & $\begin{array}{l}\text { Gegensteuernde } \\
\text { Maßnahmen, } \\
\text { Fasten und } \\
\text { Substanzmissbrauch }\end{array}$ & $\begin{array}{l}\text { Atypische } \\
\text { Essanfälle }\end{array}$ & Gesamtwert \\
\hline \multicolumn{8}{|l|}{ EDE } \\
\hline Restraint & $0,73 * *$ & $0,38 * *$ & 0,21 & $0,28 *$ & $0,69^{* *}$ & $-0,05$ & $0,61 * *$ \\
\hline Eating Concern & $0,48 * *$ & $0,54 * *$ & 0,21 & $0,61 * *$ & $0,47 * *$ & $0,31^{* *}$ & $0,72 * *$ \\
\hline Weight Concern & $0,37 * *$ & $0,44 * *$ & $0,24 *$ & $0,23 *$ & $0,35 * *$ & 0,20 & $0,48 * *$ \\
\hline Shape Concern & $0,52 * *$ & $0,56 * *$ & $0,38 * *$ & $0,27^{*}$ & $0,42 * *$ & 0,15 & $0,62 * *$ \\
\hline \multirow[t]{3}{*}{ EDE gesamt } & $0,67 * *$ & $0,61 * *$ & $0,32 * *$ & $0,45^{* *}$ & $0,62 * *$ & 0,19 & $0,77 * *$ \\
\hline & \multicolumn{7}{|l|}{ SIAB-S $(\mathrm{n}=81)$} \\
\hline & $\begin{array}{l}\text { Allgemeine } \\
\text { Psychopathologie } \\
\text { und soziale } \\
\text { Integration }\end{array}$ & $\begin{array}{l}\text { Bulimische } \\
\text { Symptome }\end{array}$ & $\begin{array}{l}\text { Körperschema } \\
\text { und Schlank- } \\
\text { heitsideal }\end{array}$ & $\begin{array}{l}\text { Sexualität und } \\
\text { Körpergewicht }\end{array}$ & $\begin{array}{l}\text { Gegensteuernde } \\
\text { Maßnahmen, } \\
\text { Fasten und } \\
\text { Substanzmissbrauch }\end{array}$ & $\begin{array}{l}\text { Atypische } \\
\text { Essanfälle }\end{array}$ & Gesamtwert \\
\hline \multicolumn{8}{|l|}{ EDE } \\
\hline Restraint & $0,43 * *$ & $0,25 *$ & $0,53 * *$ & $0,40 * *$ & $0,51 * *$ & $-0,13$ & $0,52 * *$ \\
\hline Eating Concern & $0,55 * *$ & $0,60 * *$ & $0,44 * *$ & 0,18 & $0,50^{* *}$ & $0,34 * *$ & $0,68^{* *}$ \\
\hline Weight Concern & $0,31 * *$ & 0,19 & $0,38 * *$ & 0,13 & $0,30 * *$ & 0,03 & $0,35^{* *}$ \\
\hline Shape Concern & $0,41 * *$ & 0,18 & $0,41 * *$ & $0,24 *$ & $0,34 * *$ & 0,07 & $0,44 * *$ \\
\hline EDE gesamt & $0,55 * *$ & $0,41 * *$ & $0,57 * *$ & $0,31 * *$ & $0,54 * *$ & 0,10 & $0,65^{* *}$ \\
\hline
\end{tabular}

views zu Ess-Störungen - ein ausführliches Handbuch vorhanden ist. Dies sichert bei einer angemessenen Schulung von Interviewern eine hohe Interraterreliabilität. Diese wurde bei 31 auf Video aufgenommenen Interviews geprüft. Insgesamt lagen 116 vollständige Intervieweinschätzungen von 7 Beobachtern und Interviewern vor. Sie wurden jeweils paarweise zwischen den Beurteilern verglichen. Der mittlere Wert von Gesamt- $\kappa$ (d. h. ein $\kappa$-Wert wurde für alle Fragen zusammen berechnet) betrug 0,64 für den Jetzt-Zeitpunkt und 0,63 für früher, wenn die Skalierung von 0 bis 4 zugrundegelegt wurde. Nach Rekodierung der Skalierung in 0 (Einschätzung 0 oder 1 = nicht klinisch bedeutsam) oder 1 (Einschätzung 2, 3 oder $4=$ klinisch bedeutsam) ergaben sich $\kappa$-Werte von 0,81 (jetzt) und 0,85 (früher).

Skaleninterkorrelationen: Bis auf die Skala «Atypische Essanfälle» ( $\mathrm{r}=0,07$ für jetzt und $\mathrm{r}=0,19$ für früher) korrelierten die Subskalen des SIAB-EX mit Koeffizienten zwischen 0,54 und 0,82 (jetzt) bzw. 0,62 und 0,78 (früher) hoch mit dem Gesamtwert. «Atypische Essanfälle» korrelierten auch mit anderen Subskalen sehr gering. Niedrige Korrelationen mit den anderen Skalen des SIAB-EX zeigte ebenfalls die Skala «Bulimische Symptome». Generell belegen die Interkorrelationen eine sinnvolle Skalenunterteilung der SIAB-EX-Items. Die Interkorrelationen der Subskalen des SIAB-S sind verhältnismäßig hoch, insbesondere zwischen den Skalen, die Essanfälle erfassen («Bulimische Symptome» bzw. «Atypische Essanfäl- le»; 0,75 (jetzt), bzw. 0,72 (früher)). Die Korrelationen der Einzelskalen mit dem Gesamtwert bewegen sich zwischen 0,41 und 0,87 und befinden sich damit überwiegend im höheren Bereich.

Interne Konsistenz: Für den SIAB-EX fanden sich Cronbachs $\alpha$-Koeffizienten zwischen 0,52 («Gegensteuernde Maßnahmen usw.» jetzt) und 0,93 («Bulimische Symptome» jetzt und früher, Gesamtwert früher), wobei die Koeffizienten für die Jetzt- und die Früher-Einschätzung in der Höhe vergleichbar waren. Besonders konsistent zeigten sich die Subskala «Bulimische Symptome» und der Gesamtwert $(0,92)$. Niedrigere Koeffizienten ( 0,64 früher und 0,52 jetzt) waren bei der eher heterogenen Subskala «Gegensteuernden Maßnahmen, Fasten und Substanzmissbrauch» festzustellen.

Die Koeffizienten für den SIAB-S lagen für die Früher-Einschätzung zwischen 0,69 und 0,94 und fallen damit sehr gut aus, vor allem erweisen sich die Subskalen «Körperschema», «Allgemeine Psychopathologie», sowie der Gesamtwert als besonders konsistent. Für die Jetzt-Einschätzung zeigten sich Koeffizienten zwischen 0,74 und 0,92 mit einem besonders niedrigen $\alpha(0,34)$ für die Subskala «Gegensteuerende Maßnahmen, Substanzmissbrauch, Fasten und Autoaggression». Einzelheiten zur Skaleninterkorrelation und internen Konsistenz finden sich bei Fichter und Quadflieg [1999; 2001).

Sensitivität und Spezifität des SIAB-S: 279 Patienten wiesen lifetime (Früher- und Jetzt-Zustand kombiniert) eine definierte 
Ess-Störung nach DSM-IV (Anorexia nervosa oder Bulimia nervosa) gemäß dem Experteninterview SIAB-EX auf. Aufgrund der SIAB-S-Diagnosen wurden 194 korrekt zugeordnet, während dies für 78 von 98 Personen ohne definierte EssStörung zutraf. Damit ergibt sich für das SIAB-S eine Sensitivität für definierte Ess-Störungen nach DSM-IV (Anorexia nervosa oder Bulimia nervosa) von 0,70 und eine Spezifität von 0,80 . Der positive Vorhersagewert («positive predictive value», PPV) beträgt 0,91 . Die Sensitivität des SIAB-S für Jetzt-Diagnosen war 0,52, Spezifität 0,86 und der PPV betrug für diesen Erfassungszeitpunkt 0,72. Die Früher-Werte waren 0,56 (Sensitivität), 0,82 (Spezifität) und 0,90 (PPV). Einzelheiten zur Verbesserung der prädiktiven Parameter finden sich in Fichter und Quadflieg [1999].

Weitere Analysen ergaben einen SIAB-S-Gesamtwert von 1,3 als den Grenzwert, welcher am besten Personen mit einer definierten Ess-Störung nach DSM-IV nach dem Algorithmus des SIAB-EX von solchen mit einer nicht näher bezeichneten Ess-Störung trennte (für die Früher-Ausprägung; Sensitivität 0,79, Spezifität 0,66 und PPV 0,86). Auch für die Diagnosen zum Jetzt-Zeitpunkt ergab sich als bester Trennwert ein SIAB-S-Gesamtwert von 1,3 (Sensitivität 0,73 , Spezifität 0,63 und PPV 0,58).

\section{Validität}

Konvergente Validität: Ein anderes häufig gebrauchtes strukturiertes Interview zur Erfassung von Ess-Störungen ist die Eating Disorder Examination (EDE) [Fairburn und Cooper, 1993; für diese Studie von uns übersetzt]. Die EDE umfasst 34 Items, aus denen - neben einigen Items, die ausschließlich diagnostischen Zwecken dienen - 4 aufgrund theoretischer Erwägungen zusammengestellte Subskalen gebildet werden können: «Gezügeltes Essen» («Restraint»), «Probleme mit dem Essen» («Eating Concern»), «Probleme mit der Figur» («Shape Concern») und «Probleme mit dem Gewicht» («Weight Concern»). Da die EDE nur den Jetzt-Zeitpunkt (meist die letzten 4 Wochen vor dem Interview, in Ausnahmefällen die letzten 3 Monate vor dem Interview) erfasst, wurden die Ergebnisse der EDE mit den Ergebnissen zum JetztZeitpunkt des SIAB-EX und des SIAB-S verglichen (Tab. 3). Hohe Korrelationen ergaben sich zwischen der SIAB-EX Skala «Körperschema und Schlankheitsideal» und EDE«Restraint», -«Shape Concern», sowie EDE-Gesamtwert; zwischen SIAB-EX-«Allgemeine Psychopathologie und Soziale Integration» und EDE-«Eating Concern», -«Shape Concern» und -Gesamtwert; zwischen SIAB-EX-«Bulimische Symptome» und EDE-«Eating Concern»; zwischen SIAB-EX«Gegensteuernde Maßnahmen usw.» und EDE-«Restraint» und-Gesamtwert; sowie zwischen SIAB-EX-Gesamtwert und allen Skalen des EDE, wobei die Korrelation mit EDE«Weight Concern» etwas geringer ausfällt.

Wie beim SIAB-EX ergaben sich hohe Korrelationen des SIAB-S-Gesamtwerts mit allen Skalen der EDE. Ebenso korrelierte der EDE-Gesamtwert mit nahezu allen Skalen des
SIAB-S mit Ausnahme der Skala SIAB-S-«Atypische Essanfälle», welche nur mit EDE-«Eating Concern» bedeutsam korrelierte. Vergleiche dazu auch Fichter und Quadflieg [1999; 2001].

SIAB-EX und SIAB-S wurde mit den Selbsteinschätzungsverfahren Eating Disorder Inventory (EDI-2) [Garner et al., 1983; Garner, 1991], dem Three Factor Eating Questionnaire (TFEQ) [Stunkard und Messick, 1985; deutsch: Pudel und Westenhöfer, 1989], der Hopkins Symptom Checklist (SCL90-R) [Derogatis et al., 1976], dem Beck Depressions-Inventar (BDI) [Beck et al., 1961] und der PERI-Demoralisationsskala (PERI-D) [Dohrenwend et al., 1980] verglichen. Die berichteten Zahlen beziehen sich sinnvollerweise auf den Jetzt-Zeitpunkt des SIAB (Tab. 4). Es wurde erwartet, dass die SIABEX-Skala «Körperschema und Schlankheitsideal» höhere Korrelationen mit den konzeptuell ähnlichen Skalen EDI«Drang dünn zu sein» («drive for thinness»), EDI-«Körperliche Unzufriedenheit» und TFEQ-«Kognitive Kontrolle» aufweisen. In der Tat korrelierte SIAB-EX-«Körperschema und Schlankheitsideal» mit EDI-«Drang dünn zu sein» und TFEQ-«Kognitive Kontrolle» am höchsten, die gleiche SIABEX-Skala korrelierte mit EDI-«Körperliche Unzufriedenheit» geringfügig negativ. SIAB-EX-«Allgemeine Psychopathologie» zeigte hohe Korrelationen mit den Summenwerten des BDI und der PERI-D, mit dem EDI-Gesamtwert, EDI-«Ineffektivität» und EDI-«Soziale Unsicherheit», geringere mit Ess-Störungsspezifischen Skalen und der SCL-90. Für die Skala «Bulimische Symptome» (SIAB-EX) fanden sich sehr hohe Korrelationen mit EDI-«Bulimie», TFEQ-«Enthemmung» und TFEQ-«Hunger». Die SIAB-EX-Skala «Gegensteuernde Maßnahmen, Fasten und Substanzmissbrauch» korrelierte mit EDI-«Drang dünn zu sein», EDI-«Interozeptive Wahrnehmung», TFEQ-«Kognitive Kontrolle» und dem BDI. «Atypische Essanfälle» (SIAB-EX) korrelierten mäßig mit EDI-«Bulimie», EDI-«Körperliche Unzufriedenheit» und negativ - mit TFEQ-«Kognitive Kontrolle». Stärkere Zusammenhänge waren mit TFEQ-«Enthemmung» und TFEQ«Hunger» zu erkennen.

Die Skala SIAB-S-«Allgemeine Psychopathologie und soziale Integration» wies Korrelationen zwischen 0,26 («Perfektionismus») und 0,71 («Ineffektivität») mit den Skalen des EDI auf, welche sich auf eher persönlichkeitsnahe Eigenschaften beziehen, hohe Korrelationen mit BDI und PERI-D, sowie mittlere Korrelationen mit den SCL-90-Skalen. Hohe Korrelationen fanden sich zwischen SIAB-S-«Bulimie» und EDI«Bulimie», TFEQ-«Enthemmung» und TFEQ-«Hunger», sowie zwischen SIAB-S-«Körperschema und Schlankheitsideal» und EDI-«Drang dünn zu sein», dem EDI-Gesamtwert und TFEQ-«Kognitive Kontrolle». Im Allgemeinen waren die Koeffizienten bei SIAB-S-«Sexualität und Körpergewicht» niedrig, wobei die Korrelationen mit EDI-«Asketizismus», EDI-«Ineffektivität», BDI und TFEQ-«Kognitive Kontrolle» über 0,30 lagen. SIAB-S-«Atypische Essanfälle» zeigte $\mathrm{Zu}$ sammenhänge mit TFEQ-«Enthemmung», TFEQ-«Hunger» 


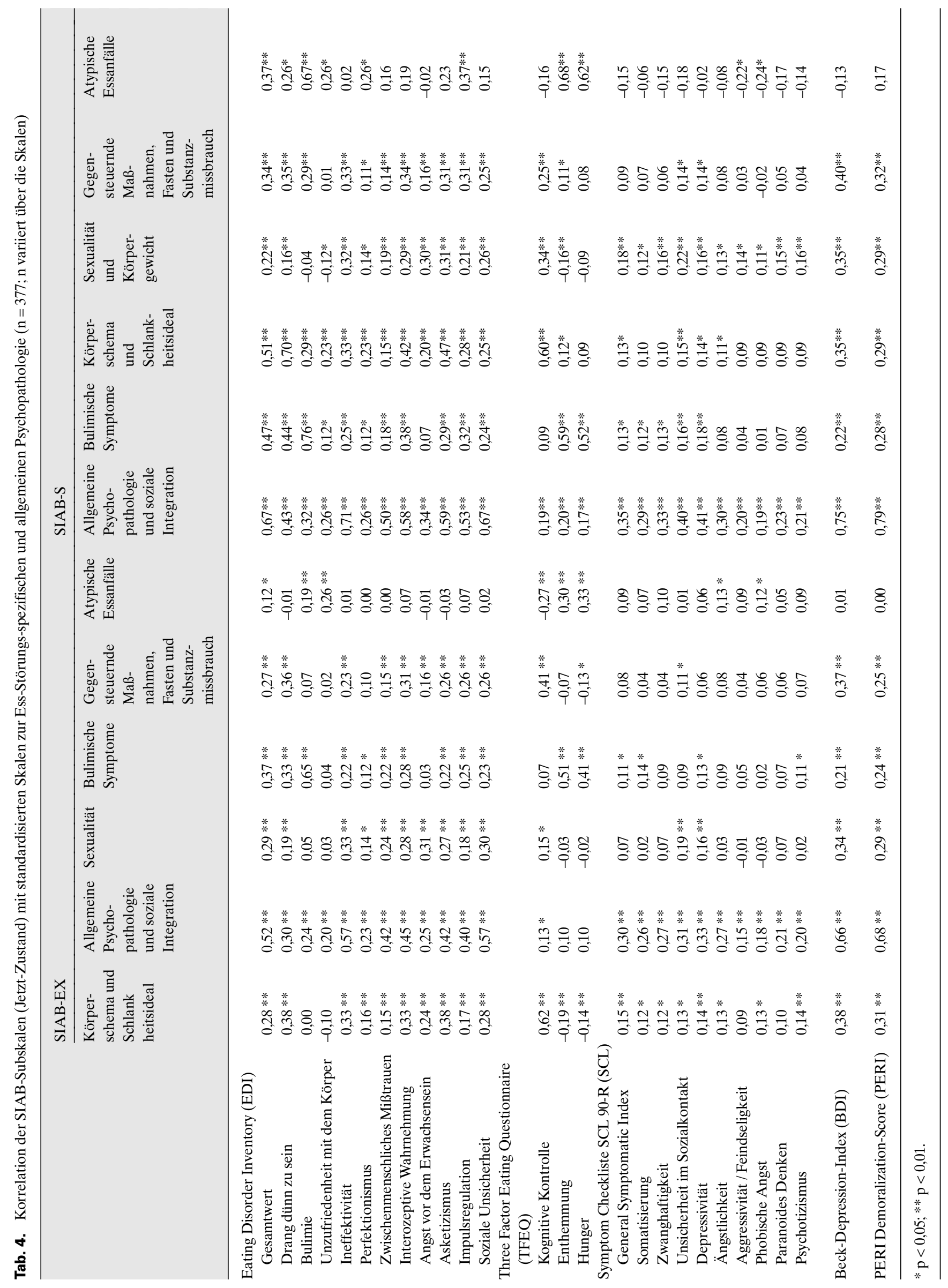


und EDI-«Bulimie». Die Korrelationen zwischen SIAB-S«Gegensteuernde Maßnahmen, Fasten und Substanzmissbrauch» und den EDI-Skalen «Drang dünn zu sein», «Ineffektivität», «Interozeptive Wahrnehmung», «Asketizismus», «Impulsregulation» und Gesamtwert sind im mittleren Bereich angesiedelt.

Diskriminante Validität: Alle Skalen der SCL-90 zeigten mit den Skalen des SIAB-EX und des SIAB-S geringe Korrelationen. Eine Ausnahme stellte die Korrelation mit SIAB-EX«Allgemeiner Psychopathologie» dar. BDI und PERI-D dagegen zeigten bedeutsame Zusammenhänge mit allen SIABEX-Skalen außer «Atypischen Essanfällen». Höhere Korrelationen fanden sich zwischen Depression bzw. Demoralisation und der allgemeinen Psychopathologie. EDI-«Bulimie» korreliert nicht mit SIAB-EX-«Körperschema und Schlankheitsideal», hingegen hoch mit SIAB-EX-«Bulimische Symptome». Die Mehrzahl der persönlichkeitsbezogenen EDI-Skalen zeigten die höchsten Korrelationen mit der «Allgemeinen Psychopathologie» (SIAB-EX). TFEQ-«Enthemmung» und TFEQ-«Hunger» korrelierten nur mit SIAB-EX-Skalen, die sich auf Essanfälle beziehen.

SIAB-S-«Allgemeine Psychopathologie und soziale Integration» sowie SIAB-S-«Bulimie» wiesen niedrige Korrelationen mit den Ess-Störungs-spezifischen Skalen des TFEQ und den Ess-Störungs-bezogenen Skalen des EDI auf. SIAB-S-«Körperschema und Schlankheitsideal» und TFEQ-«Enthemmung» sowie TFEQ-«Hunger» korrelierten gering, etwas höhere Korrelationen fanden sich zwischen dieser SIAB-S-Skala und den Skalen des EDI und dem BDI. SIAB-S-«Sexualität und Körpergewicht», SIAB-S-«Atypische Essanfälle», sowie SIAB-S- «Gegensteuernde Maßnahmen, Fasten und Substanzmissbrauch» wiesen keine bedeutsamen Korrelationen mit den anderen Skalen auf, mit Ausnahme von mittleren Korrelationen der Skala «Gegensteuerenden Maßnahmen usw.» mit BDI und PERI-D.

\section{Vergleich der Skalenprofile von Anorexia nervosa und}

Bulimia nervosa bei stationären Patienten

In den Tabellen 1 und 2 sind die Mittelwerte der Skalen des SIAB-EX, bzw. des SIAB-S bei stationär behandelten Patienten mit Ess-Störungen dargestellt. Die Mittelwerte der Selbstauskunft beruhen auf der Faktorenstruktur des SIAB-S. Zur statistischen Absicherung der Mittelwertsdifferenzen zwischen Anorexia nervosa und Bulimia nervosa wurden t-Tests gerechnet. Da jeweils Gruppen von mehreren t-Tests berechnet wurden, wurde als Schwelle ein Signifikanzniveau von 1\% zur Kontrolle multipler Testeffekte zugrundegelegt.

Für beide Messzeitpunkte ist der Gesamtwert des SIAB-EX bei Anorexia nervosa und Bulimia nervosa vergleichbar hoch, Patienten mit Anorexia nervosa weisen im Vergleich zu Patienten mit Bulimia nervosa höhere Werte in der Skala «Körperschema und Schlankheitsideal» für beide Zeitpunkte und für den Zeitpunkt der Aufnahme in die Klinik auch höhere Werte bezüglich der Störung der Sexualität auf. Patienten mit
Bulimia nervosa dagegen haben - wenig überraschend - höhere Werte in den Skalen «Bulimische Symptome» und «Atypische Essanfälle» (Tab. 1).

Auffällige Unterschiede zwischen Anorexia nervosa und Bulimia nervosa finden sich bei den SIAB-S-Skalen «Bulimische Symptome» und «Atypische Essanfälle» für beide Zeitpunkte, wobei erwartungsgemäß Bulimia-nervosa-Patienten höhere Werte aufweisen. Körperschemastörung und Schlankheitsideal sind zwischen den beiden Gruppen in der Selbstauskunft im allgemeinen nicht unterschiedlich, allerdings weisen $\mathrm{Pa}$ tienten mit Anorexia nervosa bei Aufnahme eine signifikant stärker ausgeprägte Körperschemastörung auf. Bei Aufnahme geben die magersüchtigen Patienten mehr klinisch relevante Beeinträchtigung von Sexualität und Körpergewicht an. Patienten mit Bulimia nervosa berichten für die Lebenszeit bis zur Aufnahme (früher) mehr Pathologie bei gegensteuernden Maßnahmen, Substanzmissbrauch, Fasten und Autoaggression, bei ihnen ist für den selben Zeitraum auch der Gesamtwert des SIAB-S höher als bei den Magersüchtigen.

\section{Vergleichswerte (SIAB-EX) für junge Frauen aus der Normalbevölkerung}

Tabelle 5 gibt für das Experten-Interview SIAB-EX einen Überblick über die Summenwerte von 202 jungen Frauen aus der Normalbevölkerung, die keine Ess-Störung aufwiesen. Die detaillierten Angaben zu den Einzelitems für die stationäre Patientenstichprobe sind in Fichter und Quadflieg [1999] abgedruckt.

Sämtliche Summenwerte des SIAB-EX der Frauen aus der Normalbevölkerung liegen hoch signifikant unter denen der stationären Stichprobe (t-Test, alle $\mathrm{p}<0,000)$. Die Skalen, die eine Beeinträchtigung der Sexualität erfassen, weisen die höchsten Werte von allen Skalen des SIAB-EX in der Vergleichsgruppe auf, während gegensteuernde Maßnahmen offensichtlich am seltensten vorhanden sind.

In einer weiteren Analyse sind wir der Frage nachgegangen, welcher Summenwert des SIAB-EX die Patienten der Klinik Roseneck von den Frauen aus der Bevölkerung trennt. Da in den meisten klinischen und epidemiologischen Studien das Interesse sich darauf konzentrieren dürfte, Personen, die irgendwann im Leben eine Ess-Störung hatten; zu identifizieren, wurden die Lifetime-Gesamtwerte (Maximum aus jetzt und früher) des SIAB-EX verwendet. In der Stichprobe aus der Normalbevölkerung wurden die Frauen ausgeschlossen, die in ihrem Leben eine Anorexia nervosa, Bulimia nervosa, Binge Eating Disorder, oder eine nicht näher bezeichnete EssStörung der ersten drei Beispiele des DSM-IV aufwiesen. Um die Stichprobe aus der Klinik Roseneck für diese Analyse vergleichbar zu machen, wurden in dieser Stichprobe nur die Patienten berücksichtigt, die eine der genannten Lifetime-Diagnosen aufwiesen. Dies waren 300 Patienten. Ein LifetimeGesamtwert («worst ever») von 1,0 trennt die Patienten sehr gut von den Frauen ohne Ess-Störung. Legt man einen Cutoff-Wert von größer als 1,0 fest, so ergibt sich eine Sensitivität 
Tab. 5a. SIAB-EX (früher) für Frauen ohne Ess-Störungen aus der Normalbevölkerung und Patienten mit Anorexia und Bulimia nervosa

\begin{tabular}{|c|c|c|c|c|}
\hline \multirow[b]{2}{*}{ Item No. } & & \multirow{2}{*}{$\begin{array}{l}\text { Keine Ess-Störung } \\
(\mathrm{n}=202) \\
M(\mathrm{SD})\end{array}$} & \multirow{2}{*}{$\begin{array}{l}\begin{array}{l}\text { Anorexia nervosa } \\
(\mathrm{n}=60)\end{array} \\
\mathrm{M}(\mathrm{SD})\end{array}$} & \multirow{2}{*}{$\begin{array}{l}\begin{array}{l}\text { Bulimia nervosa } \\
(\mathrm{n}=97)\end{array} \\
\mathrm{M}(\mathrm{SD})\end{array}$} \\
\hline & & & & \\
\hline & I Körperschema und Schlankheitsideal & $0,6(0,3)$ & $2,6(0,4)$ & $2,1(0,6)$ \\
\hline 16 & Körperschemastörungen & $0,6(0,8)$ & $3,4(0,8)$ & $2,4(1,2)$ \\
\hline 7 & Angst vor Gewichtszunahme & $1,0(0,9)$ & $3,6(0,6)$ & $3,0(0,8)$ \\
\hline 61 & Gedankliche Zentriertheit auf Schlankheit der Figur & $1,0(1,0)$ & $3,3(0,8)$ & $3,0(1,0)$ \\
\hline (1) & Untergewicht (modifiziert) / Abweichung vom normalen Körpergewicht & $0,6(0,7)$ & $3,9(0,4)$ & $1,7(1,4)$ \\
\hline 59 & Zwangsartige Handlungen bezüglich Essen & $0,0(0,2)$ & $2,2(1,0)$ & $1,2(1,3)$ \\
\hline 42 & Exzessive körperliche Bewegung & $1,3(1,1)$ & $2,5(1,4)$ & $2,0(1,4)$ \\
\hline 60 & Gedankliche Zentriertheit bezüglich Essen & $0,6(0,9)$ & $3,6(0,7)$ & $3,5(1,0)$ \\
\hline 14 & Ausspucken von Nahrungsmitteln & $0,0(0,1)$ & $0,9(1,3)$ & $0,8(1,3)$ \\
\hline 3 & Innere Leistungsorientierung & $1,1(1,1)$ & $2,3(1,3)$ & $1,8(1,3)$ \\
\hline 10 & Abhängigkeit des Selbstwertgefühls von Figur und Gewicht & $1,4(1,0)$ & $3,3(0,9)$ & $3,1(0,7)$ \\
\hline 13 & Kaloriengrenzen & $0,3(0,8)$ & $1,8(1,9)$ & $2,2(1,7)$ \\
\hline 19 & Amenorrhoe (modifiziert) & $0,3(0,7)$ & $2,1(0,6)$ & $1,5(1,0)$ \\
\hline 55 & Verstopfung & $0,5(0,9)$ & $2,1(1,6)$ & $2,0(1,7)$ \\
\hline 15 & Wiederkäuen & $0,0(0,0)$ & $0,2(0,7)$ & $0,3(1,0)$ \\
\hline & II Allgemeine Psychopathologie & $0,5(0,5)$ & $1,5(0,7)$ & $1,8(0,8)$ \\
\hline 66 & Vermindertes Vertrauen in eigene Fähigkeiten & $0,7(1,0)$ & $2,1(1,1)$ & $2,6(1,2)$ \\
\hline 65 & Depressive Gedanken & $0,7(1,1)$ & $2,1(1,2)$ & $2,5(1,3)$ \\
\hline 64 & Depressive Stimmung & $0,8(1,0)$ & $2,1(1,2)$ & $2,4(1,0)$ \\
\hline 67 & Verminderung des Selbstwertgefühls & $0,9(1,0)$ & $2,5(1,2)$ & $2,9(1,2)$ \\
\hline 4 & Ängste & $0,7(1,1)$ & $1,4(1,3)$ & $1,5(1,4)$ \\
\hline 6 & Schlafstörungen & $0,7(1,1)$ & $2,0(1,6)$ & $2,4(1,5)$ \\
\hline 62 & Gefühl eigener Insuffizienz und Hilflosigkeit & $0,7(1,0)$ & $2,3(1,1)$ & $2,6(1,1)$ \\
\hline 58 & Zwangsgedanken und Zwangsgrübeln & $0,2(0,6)$ & $0,5(0,9)$ & $0,6(1,0)$ \\
\hline 68 & Suizidgedanken & $0,5(0,9)$ & $2,0(1,5)$ & $2,5(1,4)$ \\
\hline 5 & Phobien & $0,6(0,8)$ & $1,1(1,2)$ & $1,4(1,1)$ \\
\hline 56 & Kontroll- oder Wiederholungszwang & $0,2(0,5)$ & $0,6(1,0)$ & $0,6(1,0)$ \\
\hline 69 & Suizidhandlungen & $0,1(0,5)$ & $0,6(1,2)$ & $1,0(1,3)$ \\
\hline 70 & Autoaggressive Verhaltensweisen & $0,1(0,5)$ & $0,8(1,2)$ & $1,1(1,4)$ \\
\hline \multirow[t]{2}{*}{57} & Sauberkeitszwänge & $0,1(0,3)$ & $0,8(0,9)$ & $0,8(1,1)$ \\
\hline & III Sexualität und Soziale Integration & $0,7(0,6)$ & $2,7(0,6)$ & $2,5(0,7)$ \\
\hline 78 & Sexuelles Verhalten & $0,2(0,7)$ & $2,6(1,7)$ & $1,8(1,8)$ \\
\hline 76 & Sexuelle Ängste & $0,5(1,1)$ & $2,8(1,4)$ & $2,3(1,5)$ \\
\hline 79 & Mangel an aktiver Freizeitgestaltung & $1,5(1,4)$ & $2,9(1,2)$ & $2,8(1,2)$ \\
\hline 82 & Kontaktvermeidung & $0,7(1,3)$ & $2,4(1,3)$ & $2,2(1,4)$ \\
\hline 81 & Qualität sozialer Kontakte & $0,4(1,0)$ & $1,6(1,6)$ & $2,2(1,8)$ \\
\hline 83 & Krankheitsverleugnung & $0,3(0,7)$ & $3,3(1,2)$ & $2,8(1,4)$ \\
\hline 84 & Globale Symptomeinschätzung & $0,5(0,7)$ & $3,8(0,4)$ & $3,7(0,5)$ \\
\hline 80 & Vermindertes Ausmaß sozialer Kontakte & $1,2(1,2)$ & $2,4(1,3)$ & $2,2(1,3)$ \\
\hline 53 & Tatsächliche Beeinträchtigung & $0,5(0,9)$ & $2,8(1,3)$ & $2,8(1,1)$ \\
\hline \multirow[t]{2}{*}{63} & Verminderte Wahrnehmung innerer Reize & $0,5(0,8)$ & $2,5(0,8)$ & $2,5(0,8)$ \\
\hline & IV Bulimische Symptome & $0,3(0,4)$ & $1,9(1,4)$ & $3,4(0,5)$ \\
\hline $21 \mathrm{a}$ & Essanfälle (objektiv) & $0,3(0,7)$ & $2,1(1,7)$ & $3,6(0,6)$ \\
\hline 23 & Häufigkeit von Essanfällen (3 Monate) & $0,2(0,6)$ & $1,8(1,6)$ & $3,7(0,6)$ \\
\hline 24 & Häufigkeit von Essanfällen ( 6 Monate) 1 & $0,2(0,5)$ & $1,8(1,4)$ & $3,5(0,7)$ \\
\hline $21 b$ & Essanfälle (subjektiv) & $0,3(0,6)$ & $2,4(1,8)$ & $3,7(0,6)$ \\
\hline 26 & Kontrollverlust & $0,2(0,5)$ & $1,9(1,2)$ & $3,0(0,7)$ \\
\hline 28 & Gefühl des Leidens und der Besorgnis im Zusammenhang mit den Essanfällen & $0,1(0,5)$ & $2,0(1,6)$ & $2,9(1,1)$ \\
\hline 35 & Erbrechen & $0,0(0,2)$ & $2,0(1,9)$ & $3,5(1,1)$ \\
\hline 34 & Zwang oder Gier zu essen & $0,9(1,0)$ & $2,1(1,4)$ & $3,2(1,0)$ \\
\hline \multirow[t]{2}{*}{22} & Zeitlicher Verlauf der Essanfälle & $0,3(0,8)$ & $1,4(1,7)$ & $3,0(1,5)$ \\
\hline & $V$ Gegensteuernde Maßnahmen, Fasten, Substanzmissbrauch & $0,2(0,2)$ & $1,0(0,5)$ & $1,2(0,6)$ \\
\hline 38 & Appetitzügler & $0,0(0,1)$ & $0,4(0,9)$ & $0,9(1,5)$ \\
\hline 37 & Diuretika & $0,0(0,2)$ & $0,3(0,9)$ & $0,4(1,1)$ \\
\hline 41 & Exzessives Fasten & $0,4(0,9)$ & $1,5(1,8)$ & $1,9(1,6)$ \\
\hline 72 & Beruhigungsmittel & $0,0(0,2)$ & $0,3(0,5)$ & $0,5(1,0)$ \\
\hline 73 & Drogen & $0,2(0,5)$ & $0,3(0,8)$ & $0,4(0,8)$ \\
\hline 36 & Laxantienabusus & $0,0(0,1)$ & $1,7(1,7)$ & $1,7(1,7)$ \\
\hline 11 & Quantitative Nahrungseinschränkung & $0,9(1,1)$ & $3,6(0,7)$ & $3,4(0,9)$ \\
\hline 71 & Alkoholmissbrauch & $0,9(0,4)$ & $1,1(0,9)$ & $1,4(1,0)$ \\
\hline 39 & Medikamente zur Steigerung der Schilddrüsenfunktion & $0,0(0,0)$ & $0,1(0,5)$ & $0,1(0,5)$ \\
\hline 43 & Einläufe & $0,0(0,0)$ & $0,1(0,3)$ & $0,2(0,8)$ \\
\hline \multirow[t]{2}{*}{44} & Brechmittel & $0,0(0,1)$ & $0,0(0,0)$ & $0,0(0,0)$ \\
\hline & VI Atypische Essanfälle & $0,4(0,6)$ & $0,4(0,7)$ & $1,6(1,0)$ \\
\hline 31 & Häufigkeit von atypischen Essanfällen (3 Monate) & $0,4(0,8)$ & $0,5(0,9)$ & $1,7(1,5)$ \\
\hline 32 & Häufigkeit von atypischen Essanfällen (6 Monate) 1 & $0,3(0,7)$ & $0,5(1,0)$ & $1,6(1,5)$ \\
\hline 29 & Atypische Essanfälle & $0,5(0,9)$ & $0,6(1,2)$ & $2,7(1,7)$ \\
\hline 33 & Vermehrtes Essen bei Stress und Belastung & $0,6(0,9)$ & $0,7(1,3)$ & $2,1(1,9)$ \\
\hline 30 & Angenehmes Völlegefühl nach Essanfällen & $0,1(0,3)$ & $0,0(0,0)$ & $0,1(0,4)$ \\
\hline & Gesamtwert & $0.5(0.3)$ & $1,9(0,4)$ & $2,0(0,5)$ \\
\hline
\end{tabular}

$\mathrm{M}=$ Mittelwert; SD = Standardabweichung. 
Tab. 5b. SIAB-EX (jetzt) für Frauen ohne Ess-Störungen aus der Normalbevölkerung und Patienten mit Anorexia und Bulimia nervosa

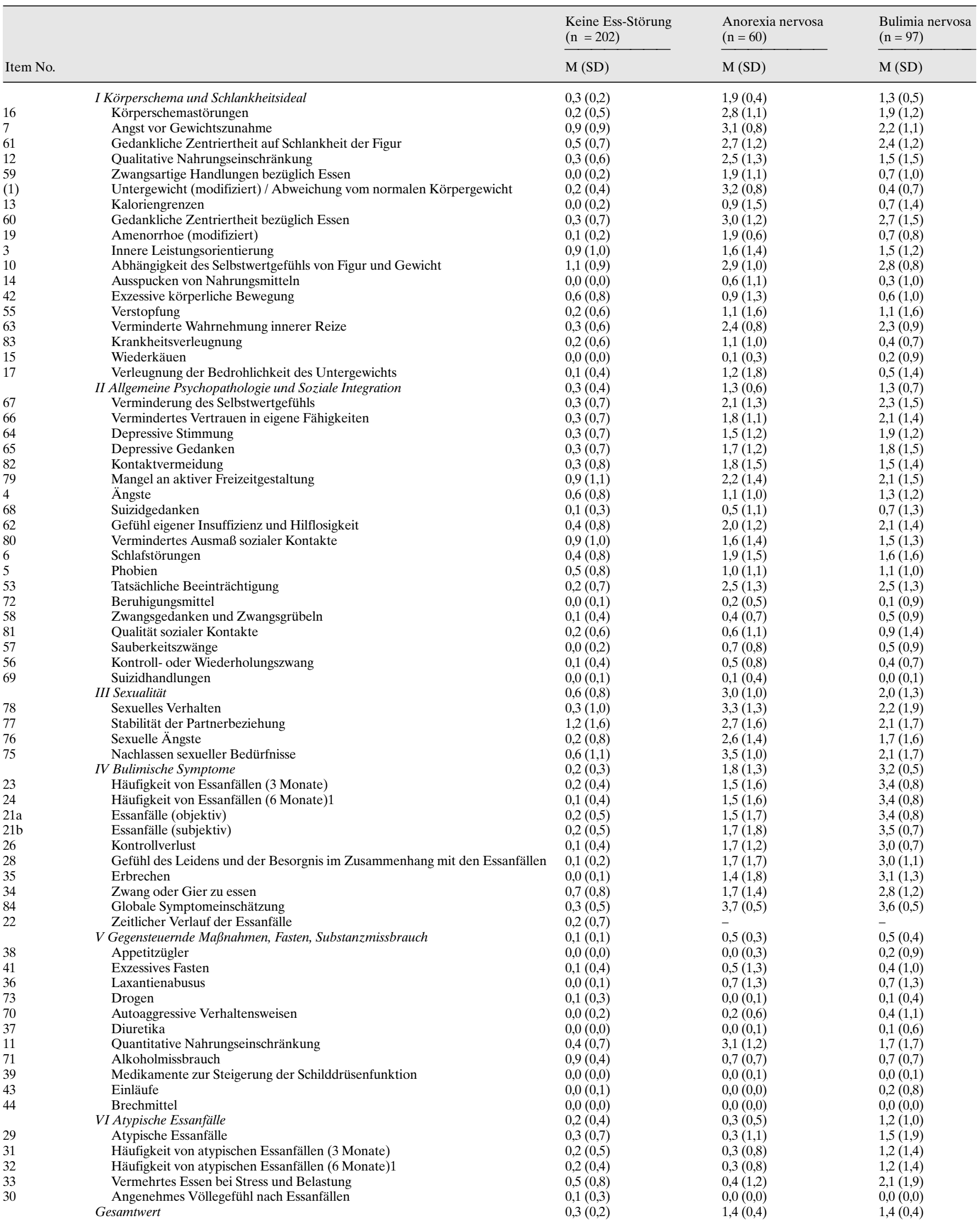

$\mathrm{M}=$ Mittelwert; SD = Standardabweichung; - = keine ausreichende Information

${ }^{1}$ Item wird zusätzlich berichtet (ist in keiner Subskala beinhaltet). 
des Lifetime-SIAB-EX-Gesamtwertes von 0,98, eine Spezifität von 0,97, sowie ein PPV von 0,98. Ein Cut-off-Wert von größer oder gleich 0,80 des Lifetime-SIAB-EX-Gesamtwertes ergibt eine Sensitivität von 1,00, eine Spezifität von 0,84 sowie einen PPV von 0,90. Eine sichere Identifikation von Personen mit einer Lifetime-Ess-Störung resultiert in einer verhältnismäßig geringen Rate falsch positiv Identifizierter von 16,3\%.

\section{Diskussion}

Während die Formulierung von diagnostischen Kriterien eine erhebliche Verbesserung und Vereinheitlichung psychiatrischer und klinisch-psychologischer Diagnose-Stellung darstellen, zeigt die Diskussion um die unterschiedlichen Ergebnisse der beiden US-amerikanischen epidemiologischen Studien «Epidemiological Catchment Area Study» (ECA) und «National Comorbidity Survey» (NCS) deutlich, dass die genaue Formulierung von Fragen im Rahmen eines klinisch-diagnostischen Interviews einen erheblichen Einfluss auf die Ergebnisse haben kann [Regier et al., 1998]. Für den SIAB-EX existieren für jede Frage in einem Manual klare Anweisungen zur Einordnung der Symptome in die Schweregradskala. Es ist das einzige auf Ess-Störungen spezialisierte Experten-Interview in deutscher Sprache, für das empirisch gewonnene Normdaten und Testgütekriterien existieren. Das SIAB-EX und das SIAB-S zeichnen sich dadurch aus, dass sie ein weites Spektrum von Ess-Störungs-typischer Psychopathologie abdecken, wobei sowohl derzeit vorhandene als auch jemals im Laufe des Lebens aufgetretene Symptome erfasst werden.

Neben quantitativen Werten wird eine solide Datenbasis für die diagnostische Beurteilung nach ICD-10 und DSM-IV bereitgestellt. Dazu ist ein Auswertungsalgorithmus zur exakten diagnostischen Einschätzung der Anorexia nervosa (restrictive type, binge eating/purging type), Bulimia nervosa und Subtypen, Binge Eating Disorder und nicht näher bezeichnete Ess-Störung erhältlich, welcher auch als SPSS-Syntax-Datei vorliegt.

Die Verfügbarkeit einer Experten- und Selbsteinschätzungsversion in Bezug auf das gleiche Item hat mehrere Vorteile. Da Selbstrating-Bögen leichter eingesetzt werden können, eignen sie sich gut als Screening-Instrument in zwei- oder mehrstufigen Verfahren, um Personen mit einem hohen Risiko für ein ausführlicheres Interview auszuwählen. Das Instrument kann für die detaillierte Einschätzung essgestörter Patienten in Längsschnittstudien über einen längeren Zeitraum verwendet werden und ebenso als Basis für die Einschätzung der Effekte psychologischer oder pharmakologischer Behandlungen auf den Verlauf der Ess-Störungen.

Zwar sind varimax-rotierte Faktoren unabhängig voneinander, doch trifft dies für die daraus gewonnenen Summenwerte in der Regel nicht zu. Die Skaleninterkorrelationen bewegten sich zwischen 0,03 und 0,55 für den SIAB-EX und zwischen 0,00 und 0,75 für den SIAB-S und zeigten damit sowohl Un- abhängigkeit als auch enge Verbundenheit. Die hohen Korrelationen fanden sich bei den Skalen, die inhaltlich von der Ess-Störungs-Symptomatik her eng verknüpft sind.

Die interne Konsistenz bewegte sich mit Cronbachs $\alpha$-Koeffizienten meist über 0,70 für beide Erfassungsmodalitäten im akzeptablen bis sehr guten Bereich [vgl. Shore und Porter, 1990]. Koeffizienten unter 0,70 weisen nur die Skalen auf, die gegensteuernde Maßnahmen mit weiteren Symptomen erfassen. Dies dürfte auf die in der Skala bereits angelegte Heterogenität der erfassten Symptome zurückzuführen sein.

Die getrennten Faktorenanalysen mit den Daten des SIABEX und den Daten des SIAB-S ergaben bemerkenswert ähnliche Faktorenstrukturen. Auffällig war die Trennung von Schlankheitsideal und Körperschemastörung bei der Selbsteinschätzung für früher, die sich nur dort fand. Die zweite auffällige Abweichung ist die Assoziation von niedrigem Körpergewicht mit Sexualität bei der Selbsteinschätzung für den Jetzt-Zeitpunkt. Bei den anderen Einschätzungen ist das niedrige Körpergewicht mit der Körperschemastörung (SIAB-S früher) bzw. Körperschema und Schlankheitsideal (SIAB-EX jetzt und früher) assoziiert. Eine ausführliche Diskussion der Ähnlichkeiten der Erfassung durch SIAB-EX und SIAB-S führen Fichter und Quadflieg [2000].

Die Konstruktvalidität des SIAB-EX und des SIAB-S wurde im Vergleich mit der international häufig verwendeten EDE überprüft. Das Ergebnis belegt eine sehr gute inhaltliche Validität beider Formen des SIAB. Ein wichtiger Unterschied ist die divergierende Definition von Essanfällen. Offensichtlich sind die Konzepte der EDE (subjektive Essanfälle, subjektives und objektives Überessen-Overeating) und die Konzepte des SIAB-EX (subjektive Essanfälle und atypische Essanfälle) nicht deckungsgleich. Eine ausführliche Diskussion dazu findet sich bei Fichter und Quadflieg [2001]. Die englischsprachige EDE ist inhaltlich sehr viel enger gefasst als das SIAB. Außerdem liegt keine offizielle deutsche Übersetzung des EDE mit überprüften Gütekriterien vor. Während das EDE ausschließlich Ess-Störungs-bezogene Symptome erfasst, beinhaltet das SIAB-EX auch zahlreiche Fragen zu nicht EssStörungs-bezogener, aber relevanter Psychopathologie. Das EDE ermöglicht die Stellung der Ess-Störungs-Diagnosen Anorexia nervosa, Bulimia nervosa und Binge Eating Disorder. Die Diagnosen Anorexia nervosa und Bulimia nervosa nach DSM-IV werden auch vom SIAB-EX erfasst, zusätzlich dazu können die im DSM-IV beschriebenen Typen dieser EssStörungen festgelegt werden. Für die Diagnose Binge Eating Disorder orientiert sich das SIAB-EX sehr eng an den im Anhang des DSM-IV beschriebenen vorläufigen Kriterien. Zusätzlich lassen sich durch das SIAB-EX sämtliche im ICD-10 (Forschungskriterien) aufgeführten Ess-Störungs-Diagnosen mittels operationalisierter Kriterien erstellen. Während das EDE - mit Ausnahme einiger diagnostischer Items, welche die letzten 3 Monate einbeziehen - lediglich die letzten 4 Wochen vor dem Interview erfasst, berücksichtigt das SIAB-EX den gegenwärtigen Zustand sowie die gesamte Lebenszeit der 
interviewten Person. Dies macht den SIAB-EX für epidemiologische Studien, klinische Diagnostik und für Therapie- und Verlaufsstudien geeignet. Vergleiche mit Selbsteinschätzungsverfahren belegen ebenfalls die gute Konstruktvalidität der SIAB-Verfahren. Sie sind allerdings mit Vorsicht zu handhaben [vgl. Steinhausen und Seidel, 1993].

In vielen klinischen Studien müssen die Teilnehmer eine volle Ess-Störungs-Diagnose nach DSM-IV oder ICD-10 aufweisen. Eine ökonomische Methode zur Identifikation potentieller Teilnehmer stellt der Fragebogen SIAB-S dar. Sensitivität, Spezifität und PPV des SIAB-S zur Unterscheidung zwischen Patienten mit einer Anorexia nervosa oder Bulimia nervosa versus Patienten mit einer nicht näher bezeichneten Ess-Störung waren - insbesondere für die Stellung von Lifetime-Diagnosen - sehr gut. In anderen Forschungssettings, wie z.B. in Studien zum Genotyp und Phänotyp von Ess-Störungen ist die sichere Identifikation von klinisch relevanten Ess-Störungen unabdingbar. Die Eignung des SIAB-EX belegen die ausgesprochen hohen Werte für Sensitivität, Spezifität und positiven Vorhersagewert bei der Unterscheidung von stationären Patienten von jungen Frauen aus der Normalbevölkerung. Diese Kennwerte liegen durchweg über $90 \%$ bei der Verwen- dung des SIAB-EX-Gesamtwertes (lifetime), der PPV z.B. bei 97,7\%. Damit beweist das SIAB-EX seine Eignung als «GoldStandard» in der standardisierten Ess-Störungs-Diagnostik. Es wurde und wird in der internationalen Multi-Center-Studie der Price Foundation zur Genetik von Ess-Störungen [Kaye et al., 2000] eingesetzt. Somit liegen auch Summenwerte des SIAB-EX von essgestörten Personen aus dem anglo-amerikanischen Sprachraum vor. Für die internationale Verwendung liegen englische, spanische und italienische Versionen vor. Die englische Fassung kann kostenlos von folgender Internetadresse heruntergeladen werden: http://www.epi.med.unimuenchen.de.

Der Vergleich von stationär behandelten Patienten mit Anorexia nervosa mit Patienten, die wegen einer Bulimia nervosa behandelt wurden, zeigte durchweg plausible Ergebnisse. Das Vorliegen höherer bulimischer Symptomatik ist bereits durch die Diagnose bedingt. Dagegen zeigten magersüchtige Patienten mehr Körperschemastörungen und ein ausgeprägteres Schlankheitsideal, sowie mehr sexuelle Ängste. Hier stimmen empirischer Befund und klinische Erfahrung weitgehend überein.

\section{Literatur}

Beck AT, Ward CH, Mendelson M, Mock J, Erbaugh J: An inventory for measuring depression. Arch Gen Psychiatry 1961;4:561-571.

Black CMD, Wilson GT: Assessment of eating disorders: Interview versus questionnaire. Int J Eat Disord 1996;20: 43-50.

Cooper Z, Fairburn C: The eating disorder examination: A semi-structured interview for the assessment of the specific psychopathology of eating disorders. Int $\mathrm{J}$ Eat Disord 1987;6:1-8.

Derogatis LR, Rickels K, Rock AF: The SCL-90 and the MMPI: A step in the validation of new self-report scale. Br J Psychiatry 1976;128:280-289.

Dilling H, Mombour W, Schmidt MH: Internationale Klassifikation psychischer Störungen, ICD-10 Kap V (F), Klinisch-diagnostische Leitlinien. Bern, Huber, 1991.

Dohrenwend BP, Shrout PE, Egri G, Mendelson FS: Nonspecific psychological distress and other dimensions of psychopathology. Arch Gen Psychiatry 1980; 37:1129-1236.

Fairburn CG, Beglin SJ: Assessment of eating disorders: Interview or self-report questionnaire? Int J Eat Disord 1994;16:363-370.

Fairburn CG, Cooper Z: The eating disorder examination, ed 12; in Fairburn CG, Wilson GT (eds): Binge Eating, Nature, Assessment, and Treatment. New York, Guilford, 1993, pp 317-360.

Fichter MM, Elton M, Engel K, Meyer AE, Mall H, Poustka F: Structured interview for anorexia and bulimia nervosa (SIAB). Development of a new instrument for the assessment of eating disorders. Int J Eat Disord 1991;10:571-592.

Fichter MM, Herpertz S, Quadflieg N, Herpertz-Dahlmann B: Structured interview for anorexic and bulimic disorders for DSM-IV and ICD-10. Updated (third) revision. Int J Eat Disord 1998;24:227-249.
Fichter MM, Quadflieg N: Strukturiertes Inventar für Anorektische und Bulimische Essstörungen (SIAB). Fragebogen (SIAB-S) und Interview (SIAB-EX) nach DSM-IV und ICD-10. Handanweisung. Göttingen, Hogrefe, 1999.

Fichter MM, Quadflieg N: Comparing self- and expert rating: A self-report screening version (SIAB-S) of the structured interview for anorexic and bulimic syndromes for DSM-IV and ICD-10 (SIAB-EX). Eur Arch Psychiatry Clin Neurosci 2000;250:175-185.

Fichter MM, Quadflieg N: The structured interview for anorexic and bulimic disorders for DSM-IV and ICD10 (SIAB-EX): Reliability and validity. Eur Psychiatry 2001;16:38-48

Garner DN, Olmstead MP, Polivy J: Development and validation of a multidimensional eating disorder inventory for anorexia nervosa and bulimia. Int J Eat Disord 1983;2:14-34.

Garner DM: Eating Disorder Inventory-2 professional manual. Odessa, Psychological Assessment Resources, 1991.

Garner DM, Garfinkel PE: The eating attitudes test: An index of the symptoms of anorexia nervosa. Psychol Med 1979;9:273-279.

Greeno CG, Marcus MD, Wing RR: Diagnosis of binge eating disorder: Discrepancies between a questionnaire and clinical interview. Int J Eat Disord 1995;17:153-160. Halmi KA, Sunday SR, Strober M, Kaplan A, Woodside DB, Fichter M, Treasure J, Berrettini WH, Kaye WH: Perfectionism in anorexia nervosa: Variation by clinical subtype, obsessionality, and pathological eating behavior. Am J Psychiatry 2000;157:1799-1805. Halmi KA, Sunday SR, Klump K, Strober M, Leckman J, Fichter M, Kaplan A, Woodside B, Treasure J, Berrettini WH, Shabbout MA, Kaye WH: Obsessions and compulsions in anorexia nervosa subtypes. Am J Psychiatry, in Druck.
Kaye WH, Lilenfeld LR, Berrettini WH, Strober M, Devlin B, Klump KL, Goldman D, Bulik CM, Halmi KA, Fichter MM, Kaplan A, Woodside DB, Treasure J, Plotnicov KH, Pollice C, Rao R, McConaha CW: A search for susceptibility loci for anorexia nervosa: Methods and sample description. Biol Psychiatry 2000;47: 794-803.

Klump KL, Bulik CM, Pollice C, Halmi KA, Fichter MM, Berrettini WH, Devlin B, Strober M, Kaplan A, Woodside DB, Treasure J, Shabbout M, Lilenfeld LR, Plotnicov KH, Kaye WH: Temperament and character in women with anorexia nervosa. J Nerv Ment Dis 2000;188:559-567.

Pudel V, Westenhöfer J: Fragebogen zum Essverhalten (FEV). Handanweisung. Göttingen, Hogrefe, 1989.

Regier DA, Kaelber CT, Rae DS, Farmer ME, Knäuper B, Kessler RC, Norquist GS: Limitations of diagnostic criteria and assessment instruments for mental disorders. Arch Gen Psychiatry 1998;55:109-115.

$\mathrm{Sa}$ H, Wittchen HU, Zaudig M: Diagnostisches und Statistisches Manual Psychischer Störungen DSM-IV. Göttingen, Hogrefe, 1996.

Shore RA, Porter JE: Normative and reliability data for 11 to 18 years olds on the eating disorder inventory. Int $\mathrm{J}$ Ea Disord 1990;9:201-207.

Steinhausen HC, Seidel R: Correspondence between the clinical assessment of eating-disordered patients and findings derived from questionnaires at follow-up. Int J Eat Disord 1993;14:367-374.

Stunkard AJ, Messick S: The three-factor eating questionnaire to measure dietary restraint, disinhibition and hunger. J Psychosom Res 1985;29:71-83. 